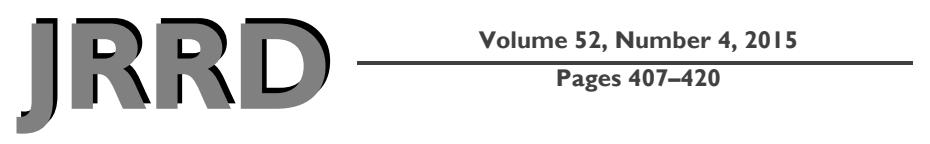

\title{
Fifteen years of experience with Integral-Leg-Prosthesis: Cohort study of artificial limb attachment system
}

\author{
Dora-Lisa Juhnke, MD; ${ }^{1}$ James P. Beck, MD; $^{2}$ Sujee Jeyapalina, PhD; ${ }^{3}$ Horst $\mathrm{H}$. Aschoff, $\mathrm{MD}^{4^{*}}$ \\ ${ }^{1}$ Klinik für Allgemein-, Gefäß-, und Viszeralchirurgie, Martin-Luther-Krankenhaus, Berlin, Germany; ${ }^{2}$ Department of \\ Orthopaedics, University of Utah School of Medicine, and Department of Veterans Affairs Salt Lake City Health Care \\ System, Salt Lake City, UT; ${ }^{3}$ Department of Bioengineering, University of Utah, Salt Lake City, UT; ${ }^{4}$ Clinic for Plastic, \\ Hand, and Reconstructive Surgery, SANA Kliniken Lübeck, Lübeck, Germany
}

\begin{abstract}
Integral-Leg-Prosthesis (ILP) is a comparatively new attachment system that allows direct skeletal docking of artificial limbs. Between January 1999 and December 2013, 69 patients with transfemoral amputation were fitted with ILPs by a single German surgeon. Device design iterations and surgical techniques evolved during these years. For the purposes of comparison, patients receiving the first two designs and procedure iterations were placed in group 1 and the patients fitted with the final design were placed in group 2. Infection rate and planned and unplanned surgical interventions were statistically compared using Fisher exact test. Data demonstrated that the high rate of stoma-associated infections seen in group 1 was dramatically reduced in group 2. Of the 39 patients with 42 implants in group 2, none had operative interventions secondary to infection. All group 2 patients remained infection-free without the use of antibiotics by following a simple but defined wound-hygiene protocol. We concluded that the final iteration of the osseointegrated intramedullary device with a low energy surface at the soft tissue and prosthesis interface allowed a biologically stable skin stoma that remained infection-free without chronic use of antibiotics. The reduction in the infection rate was attributed to the clinically based, empirically driven changes in design and surgical techniques.
\end{abstract}

Key words: above-knee amputation, amputation, device design, infection rate, Integral-Leg-Prosthesis, osseointegrated prosthesis, prosthesis, skeletal docking system, titanium niobium oxynitride surface coating, transfemoral amputation.

\section{INTRODUCTION}

The loss of a limb is associated with inescapable endstage disease or complex musculoskeletal trauma. The conventional means of exoprosthetic attachment, the centuriesold socket method of prosthetic suspension, is sometimes suboptimal, especially in cases of young and otherwise active patients with multiple amputations and/or short residual limbs. Obesity, which is further exacerbated by diminished ability to exercise, makes it difficult to fit socket suspension because of redundant fatty soft tissue. Even in patients with a normal body mass index, innovations in socket designs and liner materials are commonly unable to compensate for the intrinsically less than ideal physics and physiology of attaching an artificial limb to the vulnerable soft tissue envelope containing the remaining residual limb bone. Often, the final outcome, particularly for multiple amputations and/or short residual limbs, is accepting a life confined to a wheelchair.

\footnotetext{
Abbreviations: HIPAA = Health Insurance Portability and Accountability Act, ILP = Integral-Leg-Prosthesis, [Ti,Nb]ON = titanium niobium oxynitride.

*Address all correspondence to Horst $\mathrm{H}$. Aschoff, MD; SANA Kliniken Lübeck, Clinic for Plastic, Hand, and Reconstructive Surgery, Kronsforder Allee 71-73, 23560 Lübeck, Germany; +49 451585 1305. Email: horst.aschoff@sana.de http://dx.doi.org/10.1682/JRRD.2014.11.0280
} 
Because of these deficiencies of socket suspension, other means have been sought that would allow more functional attachment of exoprostheses to residual limbs. For over a decade, revolutionary new methods for limb attachment have been evolving in Europe [1-8]. These techniques all use various systems of percutaneous osseointegrated endoprostheses to directly attach the artificial limbs to the residual limb bone. This direct skeletal attachment obviates the need for sockets with their attendant limitations and complications [9] and has revealed other advantages such as "osseoperception" [10-11], the proprioceptive ability to feel terrain changes through the innervated residual limb. We have 15 years of experience with this new docking system, initiated in carefully selected patients with transfemoral amputations. What was initially described as the "Endo-Exo-Femurprosthesis" system is now known as the Integral-Leg-Prosthesis (ILP). Some interim clinical data have been previously published [6-8,12-15].

The purpose of this study was to find a safe and effective means to attach an artificial limb directly to the residual femur of people with transfemoral amputation. This study reports the changes in clinical outcomes during the evolution of device designs and concurrent refinement of operative techniques: three systematic and empirically driven iterations over 15 years. These changes, determined by clinical outcomes, were necessary to limit infection at the stoma and deep bone and implant interface. Because the first two design iterations and surgical procedures were quite similar and clinical outcomes were closely parallel, it was most instructive to segregate patients receiving the first two device designs (designs A and B) into group 1 and those with the latest system (design C) into group 2.

\section{MATERIALS AND METHODS}

\section{Integral-Leg-Prosthesis Design Iterations}

The group 1 implant systems had two major components: the endomodule and the skin protruding bridging connector, both made with medical grade cobalt chrome alloy. In design A (Figure 1(a)), the outer surface of the endomodule consisted of tripod-like microstructures, the Spongiosa-Metal 2 (Orthodynamics GmbH; Lübeck, Germany), intended to facilitate the osseointegration of the bone with the device. In the hope that skin would attach to the device, the bracket and the distal surface of the shaft of the prosthesis (the portion adjacent to the soft tissue interface) (Figure 1(a)) were also coated with the same rough, textured surface as the tripod-structured outer layer of the endomodule.

In the design B iteration (Figure 1(b)), the structured surface that had proved to be abrasive to the skin and soft tissues in design A was removed from the distal portion of the implant and the bracket was polished and reduced in size. Although the diameter of the bridging connector was reduced, the length of the connector from implant to stoma was maintained.

In the final device design (design C, Figure 2), the bracket was eliminated, the bridging connector was shortened to adjust to the $2 \mathrm{~cm}$-deep soft tissue channel,

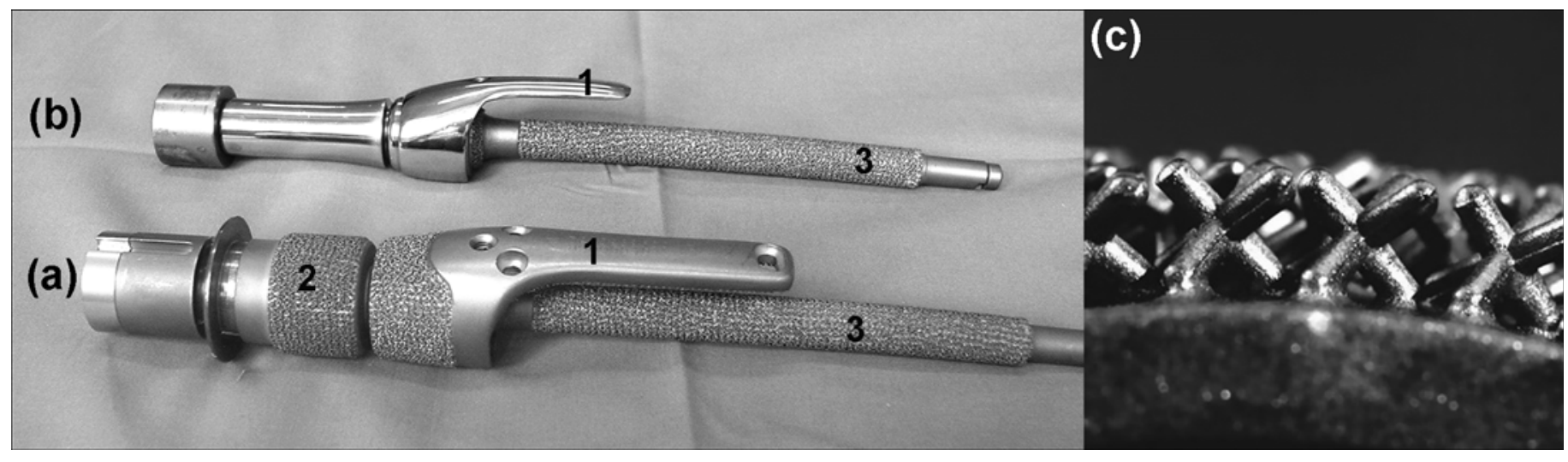

Figure 1.

Initial design iterations: (a) design A and (b) design B. In design A, portion of distal post (2) and endomodule (3) were coated with (c) Spongiosa-Metal 2 to provide structured surface for skin and bone attachment and ingrowth. Both design iterations had bonestabilizing bracket attachments (1). 


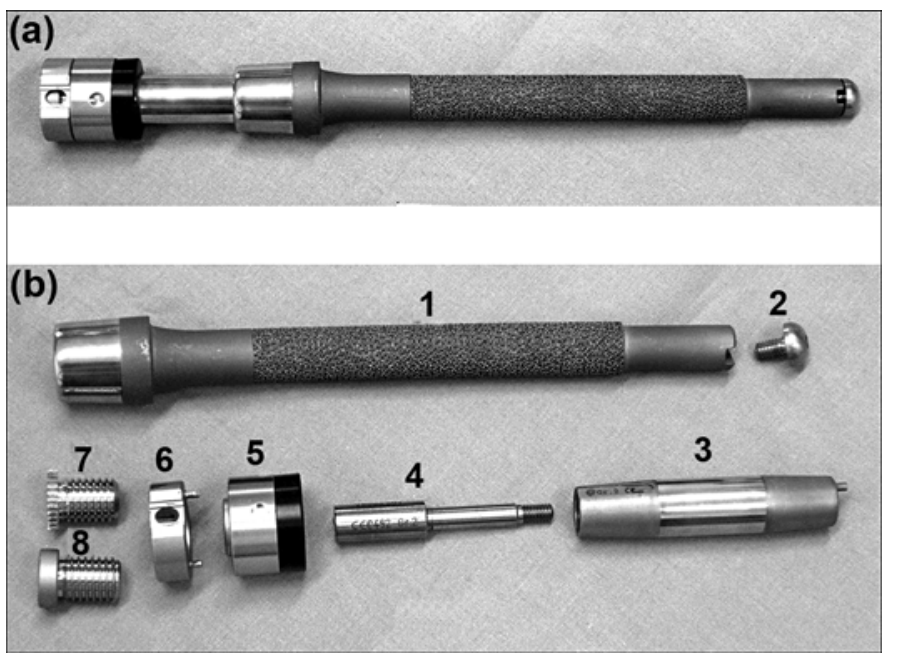

Figure 2.

Final implant iteration (design C). Implant surface that comes in contact with inner lining is coated with inert, abrasion-free surface (titanium niobium oxynitride). (a) Assembled Integral-LegProsthesis implant (design C). (b) Modular components of iteration: (1) femoral stem, (2) temporary cover screw, (3) dual cone adapter, (4) safety screw, (5) sleeve, (6) rotating disc and temporary screw (until prosthetist has made final adjustments), (7) final propeller screw, and (8) provisional screw.

and the connector as well as the bone-capping portion of the osseointegrated implant was coated with a nonabrasive titanium niobium oxynitride ([Ti,Nb]ON) ceramic (Orthodynamics GmbH).

\section{Patient Selection Criteria}

Because of specific risks inherent to the ILP attachment method, particularly the risk of infection, patients were carefully selected and were mainly those with traumatic amputations, most commonly motorcycle trauma. Inclusion criteria avoided patients with dysvascular and atrophic bone conditions and suppressed innate and humoral immunity. These conditions would be expected to limit not only an early, robust, and persistent wound healing response and osseointegration but also prevent continued protection of the stoma and the bone-implant interface over the patient's remaining life span, jeopardizing implant longevity.

Patients were assessed for the emotional stability and intelligence required to undergo rehabilitation and to understand the need for conscientious lifelong stomal wound care and hygiene. Other exclusion criteria were ongoing chemotherapy, diabetes, atherosclerotic peripheral vascular dis- ease, skeletal immaturity, and poor bone quality (e.g., bone damaged by radiation therapy, metabolic bone disease, and renal insufficiency and/or dialysis). Due to its inhibitory effect on healing, smoking was discussed as a criterion for exclusion. Because smoking is difficult to control in our population, patients were encouraged to decrease or to quit smoking altogether but were not rigorously excluded. Patients who were satisfied with conventional socket suspension technology were also excluded.

\section{Patient Consent}

All patients had a clear and explicit knowledge of the risks and benefits of the procedures prior to proposed surgeries. They underwent informed consent under the guidelines of the Ethics Committee of the SANA Kliniken Lübeck (Lübeck, Germany). The clinical study and protocols have been reviewed by the Ethics Committee of the University of Lübeck and are in compliance with the guidelines for alternative medical therapies of the World Medical Association Declaration of Helsinki. Patient record confidentiality was protected according to a protocol similar to the Health Insurance Portability and Accountability Act (HIPAA).

\section{Study Design}

From early 1999 to December 2008, all patients ( $n=$ $30)$ in clinical group 1 received either design A $(n=21$, Figure 1(a)) or subsequently design B ( $n=9$, Figure 1(b)) prostheses. It was initially assumed that a bracket was needed to prevent fatigue failure of the implant. This bracket persisted through the first two iterations but was finally discarded in device design C (Figure 2) because it added to soft tissue irritation and potentially to infection. Patients $(n=39)$ who were recruited between January 2009 and December 2013 were implanted with design C and placed in group 2 .

\section{Surgical Procedure}

Device implantation required two individually staged surgeries. The first-stage surgery involved debulking the soft tissue of the residual limb and optimizing burn surfaces and scars. After retrograde reaming of the residual femur, the endomodule was driven into the medullary canal, achieving a tight-press fit. The Morse taper at the end of the implant was capped to avoid interim ingrowth of soft tissue. In the final design (design C) and surgery iteration (group 2), thinning of the subcutaneous fat to a depth of $2 \mathrm{~cm}$ was added to the initial residual limb revision because 
it was found clinically (after the second surgery) that by decreasing the length of the channel between the skin and the bone-capping portion of the endomodule (Figure 3(b)), an advantageous rapid maturation of the granulation tissues occurred around the percutaneous portion of the device. The stage I wound was then closed, and during the interim between stages, the soft tissue wound matured and the endosteal bone grew into the implant surface.

Six weeks later, the second-stage surgery created the stoma and attached a skin-perforating bridging connector to the endomodule. The center axis of the femoral stem was located by palpation and marked with a spinal needle. A sharp circular coring cutter was then passed over the needle and the soft tissue channel was cut to the capped implant surface. Although in the design A and B protocols the diameter of this channel was kept the same as the diameter of the bridging connector, this channel in the design $C$ protocol was intentionally cut larger than the diameter of the connector shaft. This left a space of about $3 \mathrm{~mm}$ circumferentially between the shaft and the channel wall. In some early group 1 patients, a tight seal between the connector and the skin caused retained hematoma and serous fluids that contributed to failure of the implants. This $3 \mathrm{~mm}$ opening now permits gravity drainage of tissue fluids, keeping the wound clean as the stoma granulates from the depths of the wound and the epithelium migrates from the surface.

At the onset of each surgery, patients received a single $2 \mathrm{~g}$ intravenous dose of a second-generation Cephalosporin antibiotic (Biocef ${ }^{\circledR}$, PT. Otto Pharmaceutical Industries, Kuningen; Jakarta, Indonesia). Conventional means of postsurgical analgesics were also employed.

\section{Postoperative Care and Patient Satisfaction Survey}

Patients were encouraged to clean the site with mild soap and water twice a day for wound hygiene. Partial weight-bearing (i.e., crutch walking, initially 5-10 kg) and a vertical posture began immediately after the second surgery. This again promoted gravity drainage of the wound environment. Full weight-bearing without crutches usually required 4 to 6 wk from the second operation.

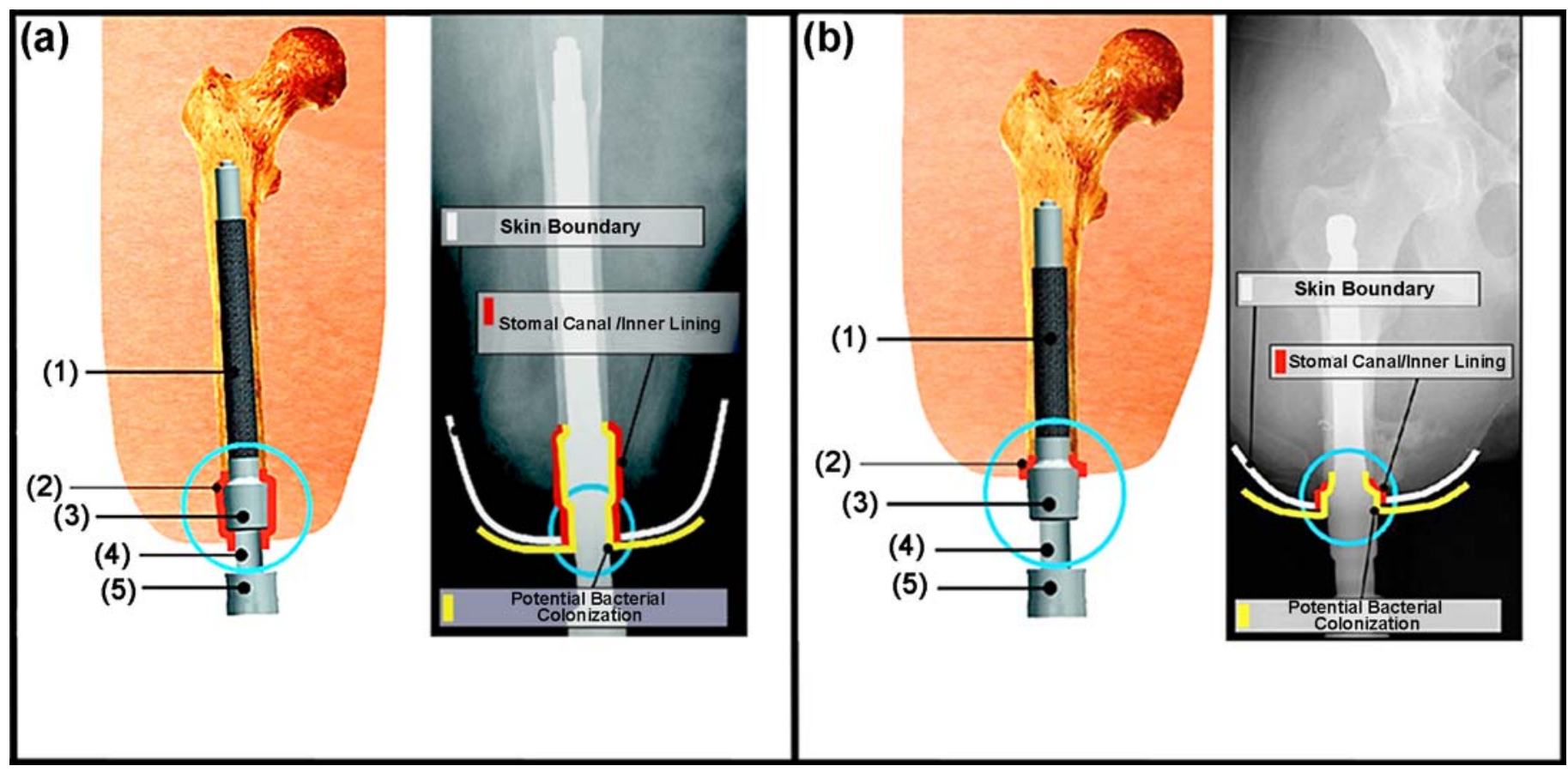

\section{Figure 3.}

Schematic representations of theoretical inner lining. (a) Deep inner lining initially tried in patients with design A and B implants, and (b) final shallow inner lining predicted in patients with design C implants. Red, white, and yellow lines represent stoma channel with epithelium (i.e., inner lining), soft tissue boundary, and sinus tract with bacterial colonization, respectively. (1) Femoral stem, (2) inner lining, (3) Morse taper, (4) dual cone adaptor, and (5) knee-connecting adaptor. Reprinted with permission from Thieme Publishers. 
Patients attended our clinic at regular intervals following the second surgery to evaluate the condition of the stoma, specifically to ensure that the interface was physiologically stable and uninfected and had reached homeostasis (Figure 4). The criteria defining homeostasis were minimal or no erythema, minimal or no drainage (some clear nonpurulent drainage was anticipated), and no pain at the stoma or bone pain during ambulation. Usually, it required about 6 wk (calculated from the second operation) for the stoma to reach homeostasis. Infection was defined as clinical evidence of stomal swelling, erythema, purulent discharge, and increasing pain.

Since all stomas were colonized by bacteria [16], the designation of "uninfected" was therefore assessed clini-

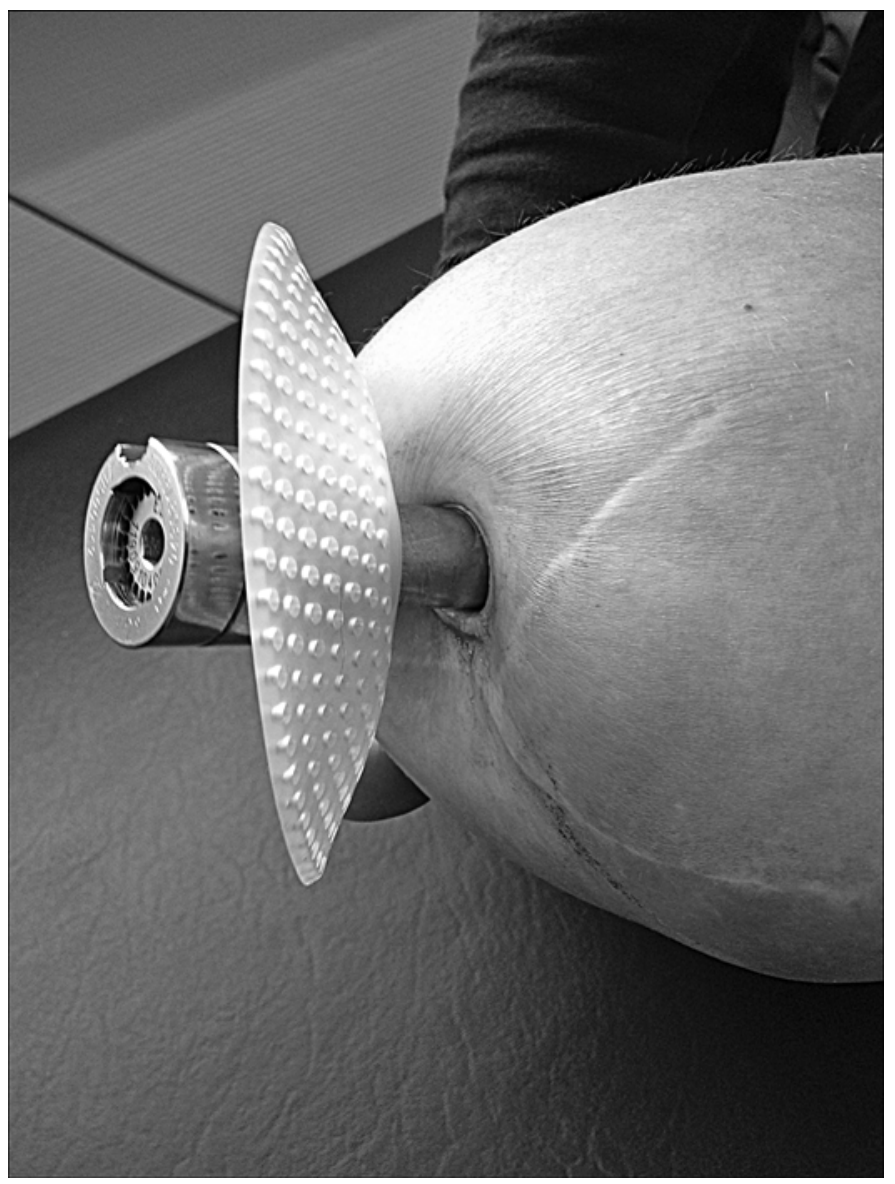

Figure 4.

Skin-implant interface showing healthy stoma with ideal homeostatic condition. Criteria defining homeostasis were (1) minimal or no erythema, (2) minimal or no drainage (some clear nonpurulent drainage is anticipated), and (3) no pain at stoma or bone pain during ambulation. cally rather than by bacterial cultures. Because the abrasive surface on design A (Figure 1(a)) irritated the skin and implant interface, a precise diagnosis of infection was problematic in group 1.

In order to guarantee adequate patient monitoring and to validate any clinical changes, a questionnaire was also designed to determine patient satisfaction with skeletal prosthetic attachment and its effect upon their quality of life. This allowed a direct comparison between their old conventional socket suspension system and their experience with the new skeletal docking system [17-18]. Criteria of performance of both suspension systems have been defined, and these data were maintained in a secured access database along with those of postoperative rehabilitation, any complications, reoperations or revisions, and operative details. The long-term goal was to create a generally accepted and reliable protocol for the use of this new method.

\section{Statistical Methods}

The data came from 73 implants in 69 patients. Implants were considered the unit of analysis $(n=73)$. Given that only 4 of the 69 patients had two implants, the potential lack of independence introduced by having two implants in 4 of the patients was intuitively negligible, so ordinary statistics were justified. The outcomes, then, were compared between the two-implant groups using a Fisher exact (two-sided comparison) test.

To verify that any lack of independence did not affect the study conclusions, a sensitivity analysis of the statistical approach was undertaken. In this analysis, one implant was dropped for each of the four patients with two implants. The dropped implant was conservatively selected to make the two groups more equivalent for each given outcome variable. Now, with one implant used per patient, independence of observations was clearly met. The conclusions of significance did not change (all $p<0.05$ remained $<0.05$ and all $p>0.05$ remained $>0.05$ ). This verified that the ordinary Fisher exact test used for the analysis provided a valid hypothesis test for the full data set (all 73 implants used).

\section{RESULTS}

Between January 1999 and December 2013, 69 patients were treated with the various iterations of the ILP, 4 of them with bilateral implants. Table 1 shows patient demographics and causes of amputations. The predominant reason for amputation was traumatic injury, usually motorcycle trauma (51 patients, 74\%) in both clinical groups. Seven patients 
Table 1.

Patient demographics and amputation characteristics.

\begin{tabular}{|c|c|c|c|}
\hline Demographics & $\begin{array}{c}\text { Designs } A \\
\text { and } B^{*}\end{array}$ & Design $C^{\dagger}$ & $p$-Value \\
\hline Amputation, $n(\%)$ & & & 0.61 \\
\hline Single-Side & $29(97)$ & $36(92)$ & \\
\hline Bilateral & $1(3)$ & $3(8)$ & \\
\hline Male, $n(\%)^{\ddagger}$ & $25(83)$ & $31(80)$ & 0.89 \\
\hline Age at Implant (yr) & & & 0.44 \\
\hline Mean \pm SD & $46 \pm 13$ & $45 \pm 12$ & \\
\hline Range & $17-69$ & $24-76$ & \\
\hline Transfemoral Amputation, $n$ (\%) & $31(100)$ & $42(100)$ & $>0.99$ \\
\hline Cause of Amputation, $n(\%)$ & & & 0.72 \\
\hline Trauma & $23(77)$ & $28(72)$ & \\
\hline Tumor & $5(17)$ & $2(5)$ & \\
\hline Infection (TEP) & $1(3)$ & $2(5)$ & \\
\hline 4th Degree Burn & $0(0)$ & $1(3)$ & \\
\hline Other & $1(3)$ & $6(15)$ & \\
\hline \multicolumn{4}{|c|}{ 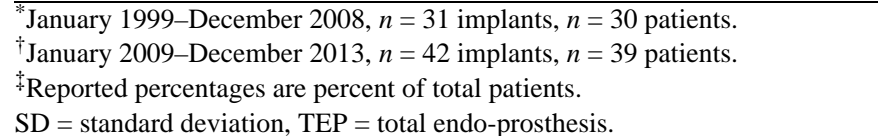 } \\
\hline
\end{tabular}

( $\sim 10 \%$ ) had single-limb amputations secondary to neoplastic disease. Three patients (4\%) had transfemoral amputations due to infected total knee arthroplasties. One patient had amputation secondary to 4th degree burn. Seven cases ( $10 \%)$ were classified as "other." The average age at time of initial amputation was $34.5 \pm 14.2 \mathrm{yr}$ (mean \pm standard deviation), with a range from 14 to $76 \mathrm{yr}$. The average age at time of implantation was $45.4 \pm 12.3 \mathrm{yr}$, with a range from 17 to 76 yr. Figure 5 shows the number of implantations performed each year.

At the conclusion of this retrospective cohort study, a total of 242 operative procedures had been done. They included 69 two-stage surgical implantation procedures (138 procedures in total) and 104 interventions (revision or unplanned intervention). It should be emphasized that one implant may account for more than one indication for interventions or revisions. Table 2 demonstrates these subgroups with a focus on the unplanned intervention rate secondary to infections and other implant failures. It is clear from this table that only group 1 patients needed reoperations or revisions due to infection. Out of 30 patients, 23 ( 77\%) in group 1 had surgical interventions secondary to infection (Table 2, Figure 6). Four of these patients $(\sim 13 \%)$ had the implant removed due to failure of bone-to-implant integrations. Two of the four patients $(\sim 6.7 \%)$ had reimplantation of ILP devices. Although there was no indication of infection, one patient had intervention to change the adapter. Thus, only 6 patients out of 30 (20\%) in group 1 did not undergo any reoperations.

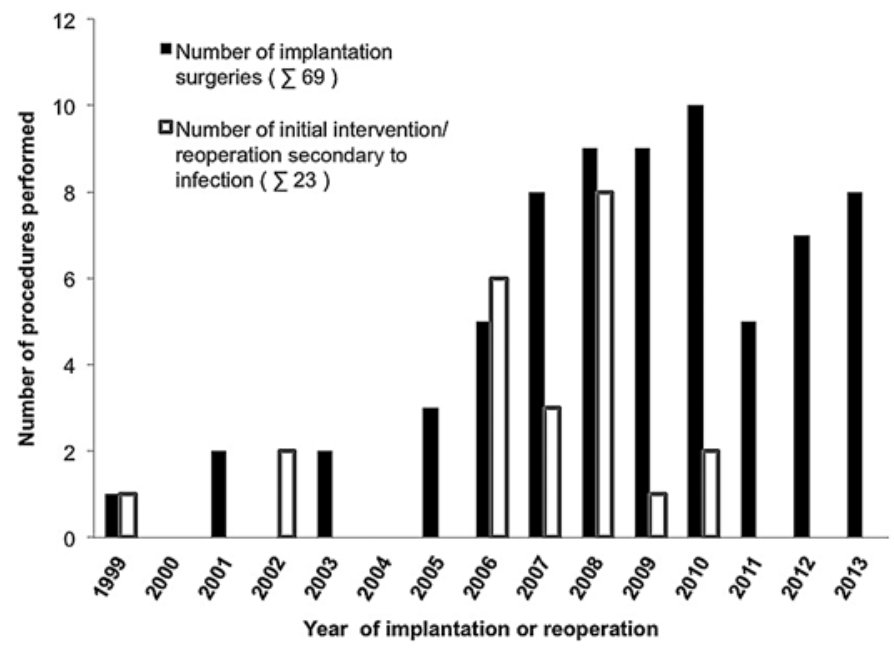

Figure 5.

Number of Integral-Leg-Prosthesis attachment surgeries and unplanned initial interventions secondary to infections performed by corresponding author between early 1999 and December 2013.

The design $\mathrm{C}$ device (group 2 patients) and the surgical modification of creating a more shallow (2 cm deep) and commodious (3 $\mathrm{mm}$ peripheral space) subcutaneous "inner lining" were introduced in January 2009. This combination appeared to significantly limit the infection and unplanned intervention rates. Out of 39 patients from group 2, 5 needed unplanned interventions at the time of this survey; this equals a reoperation rate of 12.8 percent in group 2. None of these surgeries were secondary to infections.

Reasons for interventions other than the first- or second-stage procedures have been classified into the following subgroups: early soft tissue infection, late infection of soft tissue and/or bone, structural failure of the implant, and periprosthetic or pertrochanteric fractures (Table 2). Figure 5 illustrates the total number of surgeries and the initial interventions performed secondary to infection between January 1999 and December 2013 and also shows that surgical revision of group 1 patients persisted beyond the introduction of the final iteration. As stated, more than 100 unplanned interventions in 29 patients $(n=24$ in group 1 and $n=5$ in group 2) have been undertaken. Some of these patients required one or more soft tissue or bony interventions or revisions, and these were usually early on in the group 1 patients. To date, the remaining 40 patients ( $n=6$ in group 1 and $n=34$ in group 2) have not required any unplanned intervention. 
Table 2.

Clinical outcomes (unplanned surgical interventions). Even though group 2 (design C) was 5 yr patient series, while group 1 (designs A and B) was 15 yr patient series, design C group implants remained in place (not explanted) significantly longer on average $(p=0.04)$. Design $\mathrm{C}$ reduced risk of early soft tissue infections by 42 percent compared with designs A and B (95\% confidence interval [CI]: 25\%-59\%, $p<0.001)$.

\begin{tabular}{|c|c|c|c|}
\hline Outcomes & Designs $A$ and $B^{*}$ & Design $\mathbf{C}^{\dagger}$ & $p$-Value \\
\hline $\begin{array}{l}\text { Follow-Up Time (months from implant date until either implant removal or } \\
\text { December 2013) }\end{array}$ & & & - \\
\hline Range & $6-144$ & $1-59$ & \\
\hline $\begin{array}{l}\text { Follow-Up Time (months from implant date until either implant removal or } \\
\text { December 2013), } n(\%)\end{array}$ & & & - \\
\hline $3-6$ & $1(3)$ & $3(8)$ & \\
\hline $7-12$ & $1(3)$ & $4(10)$ & \\
\hline $13-24$ & $2(7)$ & $7(18)$ & \\
\hline $25-36$ & $0(0)$ & $5(13)$ & \\
\hline Early Soft Tissue Infections (0-6 mo postimplant), $n(\%)^{\ddagger}$ & $13(42)$ & $0(0)$ & - \\
\hline Late Soft Tissue Infections (>6 mo postimplant), $n(\%)^{\S}$ & $10 / 18(55)$ & $0 / 38(0)$ & - \\
\hline Time to Late Soft Tissue Infections (mo) (>6 mo postimplant), $n(\%)^{\mathbb{q}}$ & & & - \\
\hline $7-12$ & $6(60)$ & - & \\
\hline $13-24$ & $2(20)$ & - & \\
\hline $25-36$ & $1(10)$ & - & \\
\hline $37-48$ & $1(10)$ & - & \\
\hline Structural Failure of Implant, $n(\%)$ & $1(3)^{* *}$ & - & 0.48 \\
\hline Periprosthetic or Pertrochanteric Fractures, $n(\%)$ & $3(10)^{\dagger \dagger}$ & $2(5)^{\ddagger \ddagger}$ & 0.66 \\
\hline
\end{tabular}

*January 1999-December 2008, $n=31$ implants, $n=30$ patients.

${ }^{\dagger}$ January 2009-December 2013, $n=42$ implants, $n=39$ patients.

${ }^{\ddagger}$ Out of number of implants. Absolute risk reduction: 42\% (95\% CI: 25\%-59\%, $\left.p<0.001\right)$.

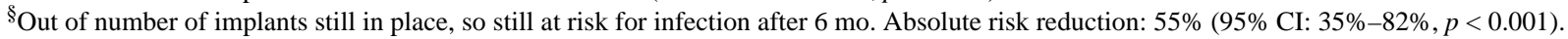

"Out of 10 late soft tissue infections.

** Occurred 7 yr after implantation.

${ }^{\dagger \dagger}$ Occurred 4, 5, and 6 mo after implantation.

勍Occurred 31 and 34 mo after implantation.

$\S \S$ Of these 4 implants, 2 could be reimplanted.

ๆศी Absolute risk reduction: 77\% (95\% CI: 51\%-84\%, $p<0.001)$.

$\mathrm{SD}=$ standard deviation.

A single intramedullary infection occurred in group 1, which could be attributed to failure to adhere to a current surgery selection criterion-exclusion for skeletal immaturity. In this particular case, primary amputation had been undertaken due to an osteosarcoma and with subsequent skeletal growth, only a thin cortical wall formed in the remaining femur. Osseointegration failed and led to oscillation of the proximal end of the implant during weightbearing. This led to infection, with implant removal 21 mo later. The surface of this explanted stem was sheathed in a substance presumed to be fibrous tissue and bacterial biofilm (Figure 7(a)). When compared with an uninfected 


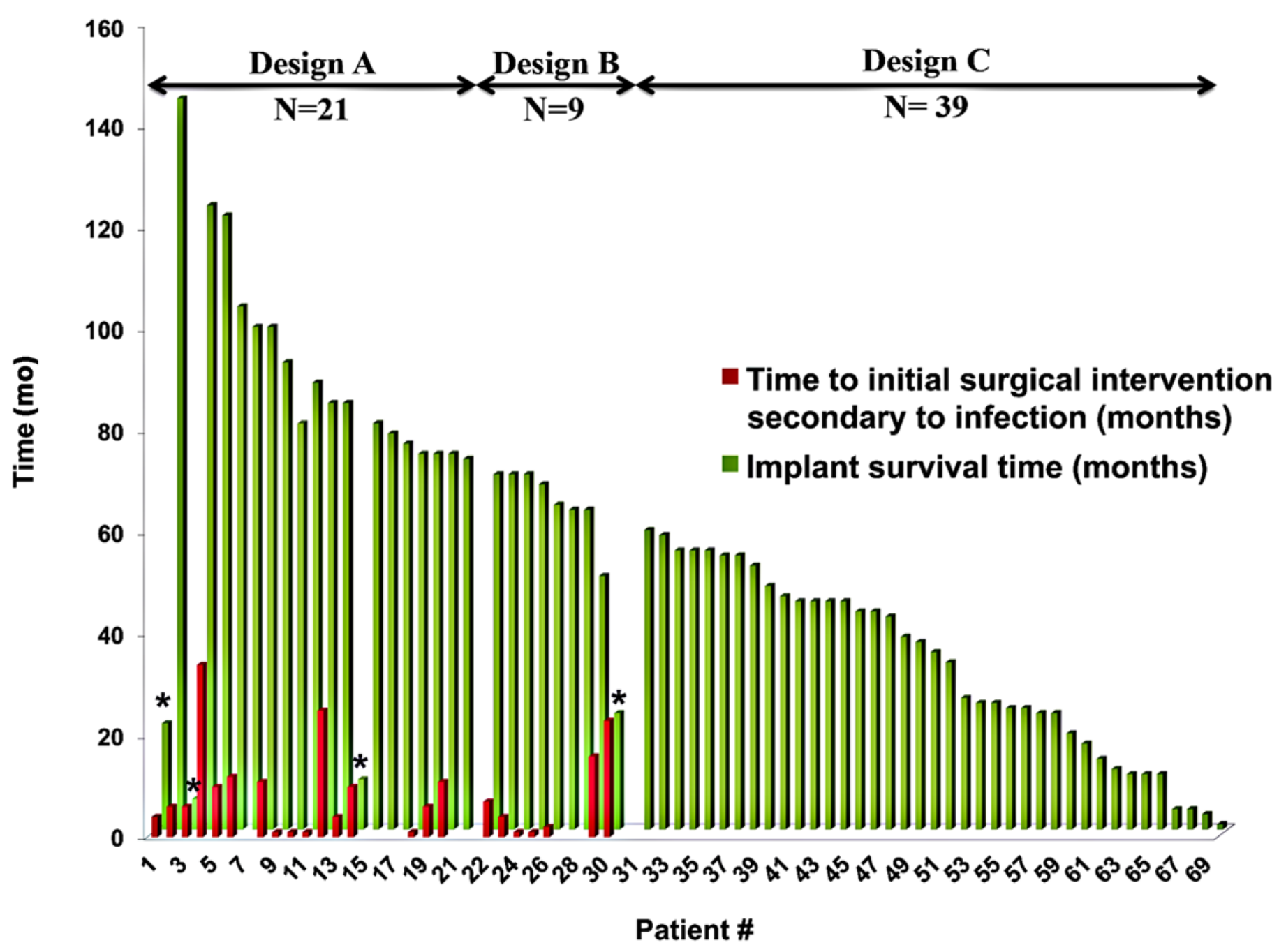

Figure 6.

Number of Integral-Leg-Prosthesis (ILP) attachment surgeries (green) and interventions secondary to infection (red) performed by corresponding author between early 1999 and December 2013. ${ }^{*}$ Total explantations of ILP with no subsequent reimplantation.

stem removed for implant material failure, the latter shows healthy cortical bone growing through the tripod structure to the implant surface (Figure 7(b)). In our opinion, the unstable mechanical situation in the osteosarcoma case prevented secure osseointegration.

Statistical analyses between the groups were undertaken, and the data (Table 2) showed significant differences between the groups (infection: $p<0.001$, surgical intervention: $p<0.001$ ). None of the group 2 patient interventions were secondary to infection. One intervention was related to soft-tissue complication $(n=1)$. Two were related to fractures, and one prolonged process sec- ondary to lengthening of the residual femur is explained later. Finally, the last case was related to revision surgery for reimplantation. The primary surgery had been carried out by a different surgeon, which resulted in nonintegration of the endomodule.

\section{DISCUSSION}

In design $\mathrm{A}$, the bracket and the distal surface of the shaft of the prosthesis were coated with a rough, textured surface. It was originally thought this might allow soft tissue 

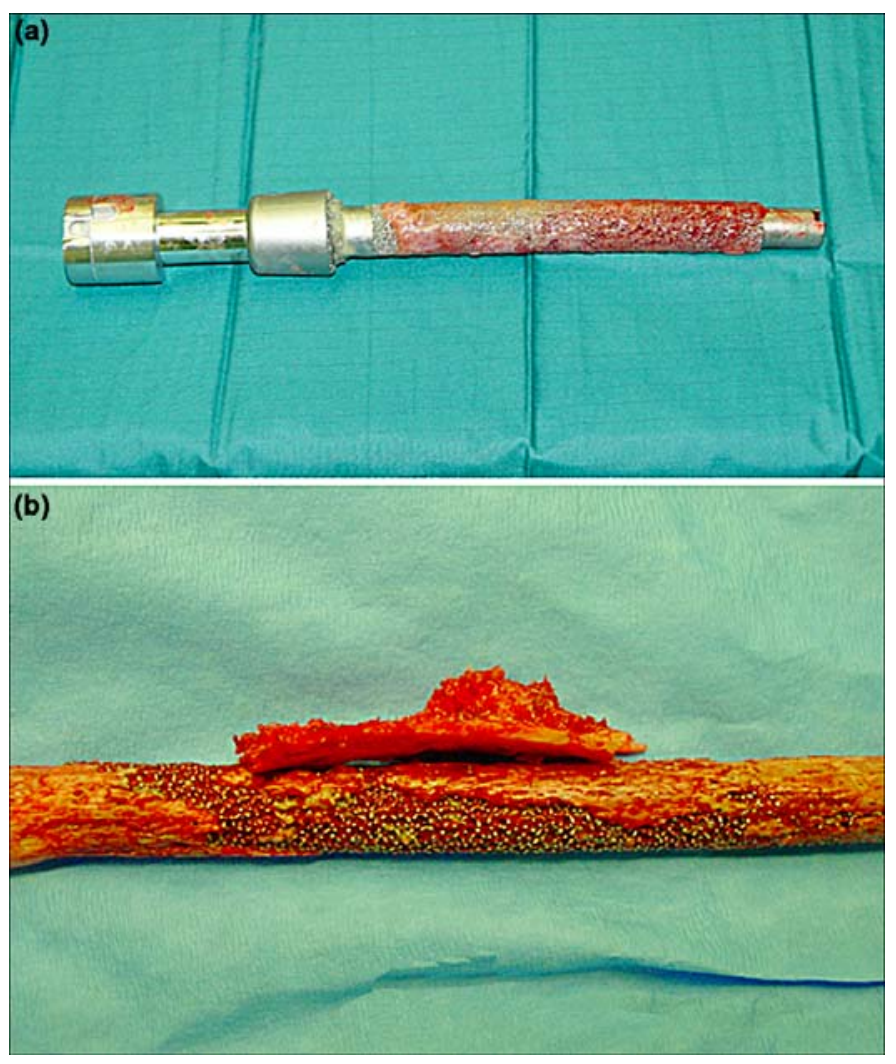

Figure 7.

Explanted endomodules from two different patients: (a) explanted stem showing no osseointegration with layer of fibrous tissue, and (b) explanted stem showing osseointegration and layer of bone.

to form a stable seal with the structured surface and create a barrier to the outside environment, preventing ascending infection. Unfortunately, this rough coating most frequently caused mechanical abrasion and irritation of the surrounding soft tissue. This continuous mechanical disruption prevented epithelial downgrowth and formation of a stable inner lining. The inner lining is defined as the surface of the adjacent soft tissues extending from the junction of the implant with the distal bone to the exit of the bridging connector from the skin (Figure 3). Although varying degrees of commensal bacterial colonization within this sinus tract was considered unavoidable and even desirable, chronic irritation and/ or subsequent superficial infection of this abraded region necessitated nearly all of the early group $1(n=23 / 30)$ revisions.

In design $\mathrm{C}$, the bone-capping portion of the osseointegrated implant was coated with a nonabrasive [Ti,Nb]ON alloy. This coating is believed to create a "lotus effect" or superhydrophobicity [19-20]. This coating prevented the undesired abrasive interaction between the soft tissue of the inner lining and the extraosseous portion of the implant. It may also establish a self-cleaning low energy surface that (along with the hygiene protocol) limits biofilm adhesion and related superficial stomal infection.

The initial problem of skin irritation within the stoma, including early and late soft tissue infections in group 1 patients, was largely eliminated by modifications in surgical technique and removing the rough structured surface. By abandoning the bracket and the abrasive surface, minimizing the inner lining tissue depth, and coating the extraosseous components with a low energy surface of [Ti,Nb]ON, no unplanned intervention secondary to infection occurred from January 2009 to December 2013. While the exact means of infection prevention is unknown, perhaps the inner lining (a superficial epithelium) grows down to and connects with the bone surface at the devicebone junction. Also, a state of homeostasis between these tissues and the low energy surface of the bridging connector allows maintenance of daily hygiene. The coating with $[\mathrm{Ti}, \mathrm{Nb}] \mathrm{ON}$, which is also used in orthopedic and dental implants, is wear- and corrosion-resistant and biocompatible [19-20]. By surface coating the implant, escape of ionic metallic oxides from the implant into the periprosthetic soft tissue is reduced, protecting patients with higher material sensitivity or allergy from unwanted foreign body reactions [19]. Wound inflammation is known to lower the $\mathrm{pH}-$ level (granulation tissue- $\mathrm{pH}$ 5.5). This acidic environment causes corrosion of commonly used implant materials. It is interesting that [Ti,Nb]ON is described in the dental literature as an especially benign tooth-implant surface for contact with the gingival mucosa [21]. This surface coating, together with daily wound hygiene, appears to prevent the formation of bacterial biofilms at the stoma.

Stable and strong bone growth into the threedimensional structured surface of the implant is absolutely essential to the success of the system. No primary ascending intramedullary infection has yet been observed in group 2 patients.

Although five patients in group 2 had unplanned intervention surgeries, none were due to infection. Periprosthetic fractures occurred in two patients. These were similar to those seen in patients with conventional endoprosthetic hip and knee replacements and cannot necessarily be linked to the ILP itself. These fractures were treated using the same techniques familiar to all arthroplasty surgeons and included dynamic hip screws (Figure 8(a)). Because our 
design C device was intentionally built (Figure 2(b)) to accept an intramedullary extension at the proximal end, this allowed an easy conversion to the configuration of a cephalomedullary nail in the event of pertrochanteric or femoral neck fracture (Figure 8(b)).

The third case that needed revision was in a patient requiring an Ilizarov procedure to gain length in the remaining proximal femoral shaft. This residual limb was simply too short to accept the length of the implant. Ultimately, the stem could be fitted into the medullary canal and weightbearing was slowly started. The radiograph of this patient (Figure 9) shows that the proximal end of the implant stem was in close proximity to the cortical surface of the greater trochanter. One week after the second-stage surgery, a fistula became evident in the region of the greater trochanter. A constant secretion of small quantities of serous fluid was also evident. Since the implant remained fixed, protected weight-bearing could be continued. The patient is now still able to walk and wears a bandage to cover the fistula and changes this dressing twice daily. Figure $\mathbf{1 0}$ shows the current status of this fistula. At the time of this review, this patient is mobile, uses a single crutch for walking balance, and is content with no further intervention.

The last two unplanned interventions were to (1) remove excess granulation tissue that formed at the

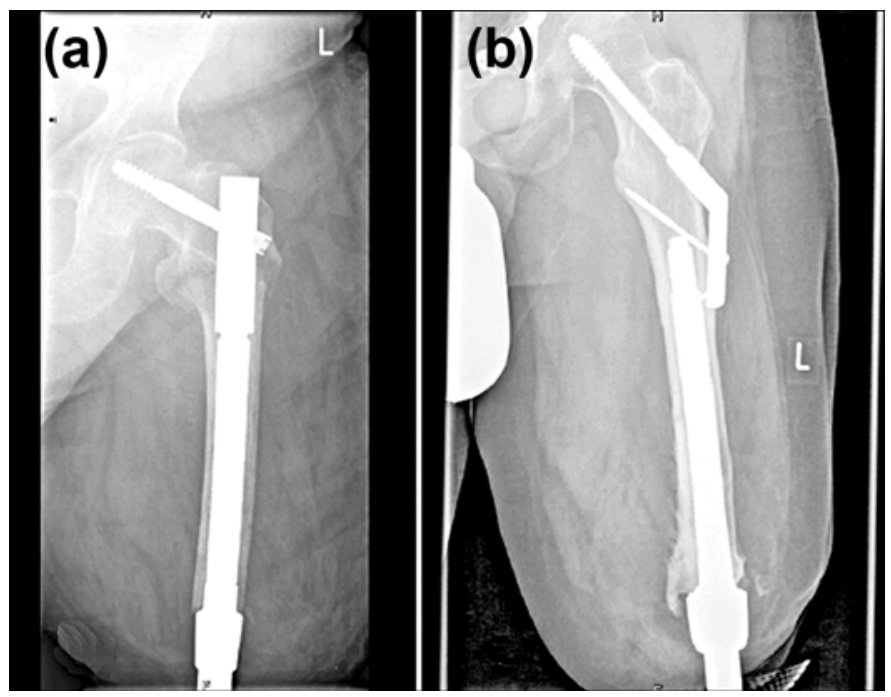

Figure 8.

Anteroposterior radiographs of postprocedural pertrochanteric bone fractures. (a) Conversion to cephalomedullary nail following pertrochanteric fracture. (b) Dynamic hip screw following pertrochanteric fracture. $L=$ left. stoma and (2) reimplant the design $\mathrm{C}$ device in a patient who underwent primary implantation by a different surgeon, which failed to osseointegrate.

While it seems intuitive that any percutaneous device that is chronically attached to bone would rapidly become infected, the entire European experience of osseointegrated prosthetic attachment has a historical long-term success rate of 70 to 80 percent $[4,8,22]$. Patients with these devices live for years with infection-free, skeletally attached artificial limbs; significantly improved activity levels; and a high quality of life [5,7,9-10,18,23-24]. This represents a remarkable achievement, particularly in patients with short residual limbs or multiple limb amputations. Understandably, the persistent 18 to 56 percent rate of infection has in the past limited the worldwide acceptance of this technology [2,22,25]. Through empirically determined but clinically derived prosthetic design and surgical technique changes and implementation of longestablished surgical principles of wound care, we feel that we have significantly improved infection control using the design $C$ device (initial absolute risk reduction of $42 \%$ and

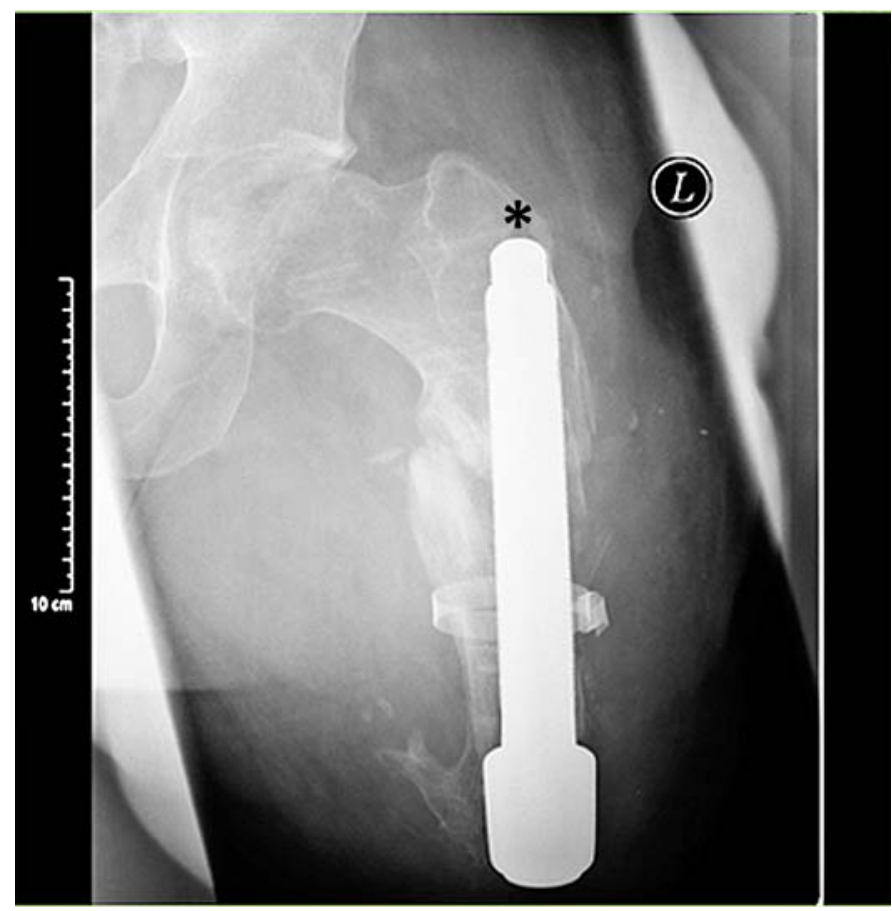

Figure 9.

Anteroposterior radiograph of Integral-Leg-Prosthesis showing proximal part of endomodule in close relation to cortical bone, greater trochanter $\left(^{\star}\right) . L=$ left. 


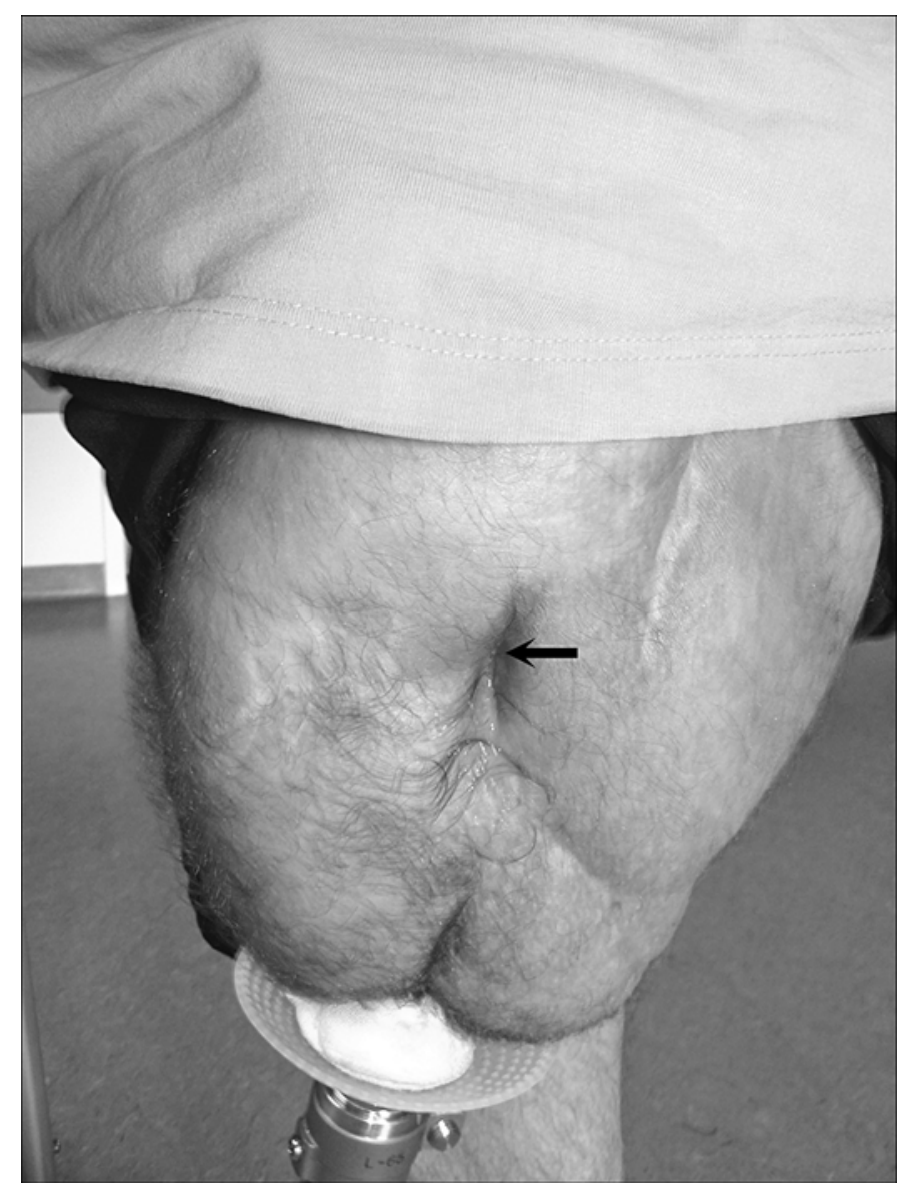

Figure 10.

Lateral view of patient (Figure 9) with chronic fistula at proximal implant level.

late absolute risk reduction of 55\%). At the conclusion of this cohort study, the infection rate following implantation of the design $C$ device remains 0 percent (absolute risk reduction of 97\%). We are now able to more safely attach artificial limbs to bone.

We have not quantified the improvement in patients' gait patterns or accessed energy consumption. Osseoperception, a result of inherent bone-proprioception, is a highly visible advantage also not yet quantified. It is likely that this technology may be cost effective over a life span. For example, the purchase and refitting costs of conventional socket suspension systems are reported to be in the range of $\$ 6,203$ to $\$ 20,070$ over the first 5 yr after primary amputation [26]. Cost analyses have shown that by avoiding multiple and expensive socket fitting and refitting, skeletal docking systems can reduce overall cost.
Looking at our long-term and recent clinical results in patients with failed conventional socket suspension of prosthetic limbs, we conclude that the early high rate of reoperation $[6,8,14]$, which was seen with the initial design iterations, is not a valid criterion for abandoning skeletal prosthetic docking. Through implant design and surgical technique changes, we strongly believe that an artificial limb that is attached directly to bone obviates the major problems of the centuries-old socket suspension systems. If a person is able to walk for $10 \mathrm{yr}$ after having received an ILP, this individual, in our experience, requests a similar skeletal docking system when encountering the need for revision. This situation is largely analogous to that of revision surgery for a worn or aseptically loosened or infected total joint arthroplasty. If the alternative to skeletal limb attachment is a life bound to a wheelchair or experiencing continuous socket pain when sitting or when walking is attempted, the risk of the occasional, largely remediable, side effects of this system of prosthetic attachment is acceptable to both patient and surgeon.

The main limitation of this study is that it was not a prospective double-blinded randomized clinical trial of a single device. This situation was not possible because of the empirical and iterative nature of the evolution of the device designs and operative technique modifications. In the future, randomized control trials are encouraged.

\section{CONCLUSIONS}

Since changes and improvements of designs and procedures have brought forth a dramatic amelioration of the initial problem of infection at the skin and implant interface, we feel the osseointegrated ILP can now be considered as an alternative method for the rehabilitation of people with amputation. Disadvantages of conventional socket suspension can be obviated with osseointegrated fixation and a skin-perforating force carrier. Solutions to the exclusion of patients with atherosclerosis and diabetes mellitus, the most common reasons for primary amputation, must ultimately be overcome to achieve the greatest application of this system. Hopefully, advances in the science of wound healing will in the future allow this broader application.

\section{ACKNOWLEDGMENTS}

\section{Author Contributions:}

Study concept and design: H. H. Aschoff.

Acquisition of data: D. Juhnke, H. H. Aschoff. 
Analysis and interpretation of data: D. Juhnke, J. P. Beck, S. Jeyapalina, H. H. Aschoff.

Drafting of manuscript: D. Juhnke, J. P. Beck, S. Jeyapalina,

H. H. Aschoff.

Critical revision of manuscript for important intellectual content:

D. Juhnke, J. P. Beck, S. Jeyapalina, H. H. Aschoff.

Statistical analysis: S. Jeyapalina.

Financial Disclosures: The authors have declared that no competing interests exist.

Funding/Support: This material was unfunded at the time of manuscript preparation.

Additional Contributions: The authors gratefully acknowledge Greg Stoddard, Division of Epidemiology, University of Utah, for his invaluable data analysis and statistical help. They also give their sincere gratitude to Gwenevere Shaw for donating her time and expertise in helping with the editing and formatting of this manuscript.

Institutional Review: Patients underwent informed consent under the guidelines of the Ethics Committee of the SANA Kliniken Lübeck (Lübeck, Germany). The clinical study and protocols have been reviewed by the Ethics Committee, University of Lübeck, and are in compliance with the guidelines for alternative medical therapies of the World Medical Association Declaration of Helsinki. Patient record confidentiality was protected according to a protocol similar to HIPAA.

Participant Follow-Up: The authors will inform the participants of the publication of this article.

\section{REFERENCES}

1. Van de Meent H, Hopman MT, Frölke JP. Walking ability and quality of life in subjects with transfemoral amputation: A comparison of osseointegration with socket prostheses. Arch Phys Med Rehabil. 2013;94(11):2174-78. [PMID:23774380] http://dx.doi.org/10.1016/j.apmr.2013.05.020

2. Brånemark R, Berlin O, Hagberg K, Bergh P, Gunterberg B, Rydevik B. A novel osseointegrated percutaneous prosthetic system for the treatment of patients with transfemoral amputation: A prospective study of 51 patients. Bone Joint J. 2014;96-B(1):106-13. [PMID:24395320] http://dx.doi.org/10.1302/0301-620X.96B1.31905

3. Kang NV, Pendegrass C, Marks L, Blunn G. Osseocutaneous integration of an intraosseous transcutaneous amputation prosthesis implant used for reconstruction of a transhumeral amputee: Case report. J Hand Surg Am. 2010;35(7):1130-34. [PMID:20541327]

4. Hagberg K, Brånemark R. One hundred patients treated with osseointegrated transfemoral amputation prosthesesrehabilitation perspective. J Rehabil Res Dev. 2009;46(3): 331-44. [PMID:19675986] http://dx.doi.org/10.1682/JRRD.2008.06.0080

5. Hagberg K, Hansson E, Brånemark R. Outcome of percutaneous osseointegrated prostheses for patients with unilateral transfemoral amputation at two-year follow-up. Arch
Phys Med Rehabil. 2014;95(11):2120-27.

[PMID:25064778]

http://dx.doi.org/10.1016/j.apmr.2014.07.009

6. Aschoff HH, Juhnke DL. [Evaluation of 10 years experience with endo-exo femur prostheses - background, data and results]. Z Orthop Unfall. 2012;150(6):607-14. German. [PMID:23171987]

7. Aschoff HH, Clausen A, Hoffmeister T. [The endo-exo femur prosthesis-a new concept of bone-guided, prosthetic rehabilitation following above-knee amputation]. Z Orthop Unfall. 2009;147(5):610-15. German. [PMID:19938359]

8. Aschoff HH, Kennon RE, Keggi JM, Rubin LE. Transcutaneous, distal femoral, intramedullary attachment for abovethe-knee prostheses: An endo-exo device. J Bone Joint Surg Am. 2010;92(Suppl 2):180-86. [PMID:21123601] http://dx.doi.org/10.2106/JBJS.J.00806

9. Hagberg K, Häggström E, Uden M, Brånemark R. Socket versus bone-anchored trans-femoral prostheses: Hip range of motion and sitting comfort. Prosthet Orthot Int. 2005; 29(2):153-63. [PMID:16281724] http://dx.doi.org/10.1080/03093640500238014

10. Hagberg K, Häggström E, Jönsson S, Rydevik B, Brånemark R. Osseoperception and osseointegrated prosthetic limbs. In: Gallagher P, Desmond D, MacLachlan M, editors. Psychoprosthetics. London (England): Springer; 2008. p. 131-40.

11. Rydevik B, Shubayev VI, Myers RR. Osseoperception. In: Brånemark $\mathrm{P}$, editor. The osseointegration book: From calvarium to calcaneus. Berlin (Germany): Quintessence; 2005. p. 149-56.

12. Aschoff HH. The endo-exo-femoral prosthesis. Traum Orthop Surg Russia. 2011;1(59):101-5.

13. Aschoff HH, Clausen A, Tsoumpris K, Hoffmeister T. [Implantation of the endo-exo femur prosthesis to improve the mobility of amputees]. Oper Orthop Traumatol. 2011;23(5):462-72. German. [PMID:22083046] http://dx.doi.org/10.1007/s00064-011-0054-6

14. Aschoff HH, et al. Die endo-exo-femurprostheses zur rehabilitation nach oberschenkelamputation. CHAZ. 2009;10: 1-6. German.

15. Rubin L, Kennon R, Keggi J, Aschoff HH. Surgical management of transfemoral amputations with a transcutaneous, press-fit distal intra-medullary device: Analysis with minimum 2 year follow-up. J Bone Joint Surg Br. 2012; 94-B(Suppl XXI):95.

16. Clausen A, Aschoff HH. Microbiology of the endo-exofemur prosthesis (EEFP) experience. Proceedings of the 1st International Endo-Exo Meeting; 2009 May; Lübeck, Germany.

17. Büll O. Theoretische Aspekte und erste praktische Ergebnisse von perkutanen Exoprothesen bei Oberschenkelamputationen. In: Medizinische fakultät. Munchen (Germany): Ludwig-Maximilians-Universitat Munchen; 2006. German. 
18. Juhnke DL. Über die Endo-Exo-Femurprothese: Erarbeitung von Konzepten für die Qualitätssicherung knochengeführter Oberschenkelprothesen durch ganganalytische Bestimmungen und Auswertung von Druckverläufen unter dem Fuß. In: Medizinische fakultät. Munchen (Germany): LudwigMaximilians-Universitat Munchen; 2006. German.

19. Thull R, Handke KD, Karle E. [Examination of titanium coated with (Ti,Nb)ON and (Ti,Zr)O in an animal experiment]. Biomed Technik. 1995;40(10):289-95. German. http://dx.doi.org/10.1515/bmte.1995.40.10.289

20. Probst J, Gbureck U, Thull R. Binary nitride and oxynitride PVD coatings on titanium for biomedical applications. Surf Coat Technol. 2001;148(2-3):226-33. http://dx.doi.org/10.1016/S0257-8972(01)01357-3

21. Nickenig HJ, Schlegel KA, Wichmann M, Eitner S. Expression of interleukin 6 and tumor necrosis factor alpha in soft tissue over ceramic and metal implant materials before uncovering: A clinical pilot study. Int J Oral Maxillofac Implants. 2012;27(3):671-76. [PMID:22616062]

22. Tillander J, Hagberg K, Hagberg L, Brånemark R. Osseointegrated titanium implants for limb prostheses attachments: Infectious complications. Clin Orthop Relat Res. 2010; 468(10):2781-88. [PMID:20473597] http://dx.doi.org/10.1007/s11999-010-1370-0

23. Hagberg K, Brånemark R, Gunterberg B, Rydevik B. Osseointegrated trans-femoral amputation prostheses: Prospective results of general and condition-specific quality of life in 18 patients at 2-year follow-up. Prosthet Orthot Int. 2008;32(1):29-41. [PMID:18330803] http://dx.doi.org/10.1080/03093640701553922

24. Hagberg K, Brånemark R, Hägg O. Questionnaire for Persons with a Transfemoral Amputation (Q-TFA): Initial validity and reliability of a new outcome measure. J Reha- bil Res Dev. 2004;41(5):695-706. [PMID:15558399]

http://dx.doi.org/10.1682/JRRD.2003.11.0167

25. Sullivan J, Uden M, Robinson KP, Sooriakumaran S. Rehabilitation of the trans-femoral amputee with an osseointegrated prosthesis: The United Kingdom experience. Prosthet Orthot Int. 2003;27(2):114-20. [PMID:14571941] http://dx.doi.org/10.1080/03093640308726667

26. Smith DG, Horn P, Malchow D, Boone DA, Reiber GE, Hansen ST Jr. Prosthetic history, prosthetic charges, and functional outcome of the isolated, traumatic below-knee amputee. J Trauma. 1995;38(1):44-47. [PMID:7745656] http://dx.doi.org/10.1097/00005373-199501000-00013

Submitted for publication November 11, 2014. Accepted in revised form March 9, 2015.

This article and any supplementary material should be cited as follows:

Juhnke D, Beck JP, Jeyapalina S, Aschoff HH. Fifteen years of experience with Integral-Leg-Prosthesis: Cohort study of artificial limb attachment system. J Rehabil Res Dev. 2015;52(4):407-20. http://dx.doi.org/10.1682/JRRD.2014.11.0280

ResearcherID: James P. Beck, MD: F-9407-2015; Sujee Jeyapalina, PhD: F-9375-2015; Horst H. Aschoff, MD: F-9751-2015

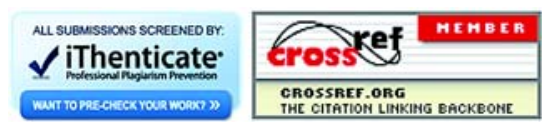


